\author{
CONVEX AND FRACTAL GEOMETRY \\ BANACH CENTER PUBLICATIONS, VOLUME 84 \\ INSTITUTE OF MATHEMATICS \\ POLISH ACADEMY OF SCIENCES \\ WARSZAWA 2009
}

\title{
FRACTAL STAR BODIES
}

\author{
IRMINA HERBURT \\ Faculty of Mathematics and Information Science, Warsaw University of Technology \\ Pl. Politechniki 1, 00-661 Warszawa, Poland \\ E-mail: herbir@mini.pw.edu.pl \\ MARIA MOSZYŃSKA \\ Institute of Mathematics, University of Warsaw \\ Banacha 2, 02-097 Warszawa, Poland \\ E-mail:mariamos@mimuw.edu.pl \\ DORETTE PRONK \\ Department of Mathematics and Statistics, Dalhousie University \\ Halifax, NS, B3H 3J5, Canada \\ E-mail: pronk@mathstat.dal.ca
}

\begin{abstract}
Since the geometry of star bodies can be considered as a part of convex geometry, it is natural to think of fractal star bodies (that is, star bodies with fractal boundaries) as objects on the border of convex and fractal geometry.

We introduce the notion of fractal star body and study some of its basic properties. We study several operations on star bodies which preserve the family of fractal star bodies.

Finally we show how the requirement that the boundary of the star body be fractal limits the possible values of the topological dimension of its kernel.
\end{abstract}

Introduction. Convex geometry and fractal geometry study objects of a fairly different nature. Generally, convex sets and their relatives are characterized by their nice

2000 Mathematics Subject Classification: 52A30, 28A80.

Key words and phrases: fractals, star bodies.

We would like to thank Marta Szumańska for her valuable remarks on an earlier version of this paper. We would also like to thank Robert Dawson for his help in creating the images for Example 3.8 .

Research of the third author was supported by NSERC and a sabbatical leave grant from Dalhousie University. She also thanks both Warsaw University and Warsaw University of Technology for their hospitality and financial support during her visit to work on this project.

The paper is in final form and no version of it will be published elsewhere. 
properties, whereas fractals are characterized by their complicated nature. However, the techniques that one uses to study these objects have a significant amount of overlap. Both streams of geometry have made extensive use of notions from measure theory and topology. The role of the measure theory in fractal geometry is obvious (see for example [5]), we just mention its role in the determination of the dimension of a fractal set and in the description of its local structure. The interaction of measure theory with convex geometry can be seen, for example, in Sections 2.2 and 2.3 of [18] concerning the boundary structure of convex bodies and in the article [19] on curvature and surface area measures.

The role of topology in convex geometry can be seen, for example, in the survey [22] and in the article [8] on Baire categories in convexity. In recent work on fractals various authors ([16] and [21] among others) have used $\varepsilon$-neighbourhoods to study their persistent homology groups and obtain new fractal invariants.

These observations have led us to ask whether there are any geometric objects that would be of interest to both fractal and convex geometers. In the study of these objects there would be natural questions coming from both types of geometry, and hopefully, the answers to these questions may lead to new constructions that could be beneficial to either or both branches of geometry.

Convex bodies would obviously not fit this description since the fact that they are convex implies that they are not fractal by any of the obvious definitions. However, star-shaped sets form a natural extension of the class of convex sets and can be required to have a fractal boundary, which would make them of interest both to people working in convex geometry and to people working in fractal geometry.

In this paper, we introduce the notion of fractal star body as a star body with a fractal boundary. For our notion of fractal we will use the dimension criterion, namely that the fractal dimension of an object is greater than its topological dimension, for some notion of fractal dimension. There are other criterions one may choose to define the notion of being fractal as mentioned for example in [5], nowhere-differentiability being one of them. However, the dimension criterion singles out a more distinctive class of objects, as was shown for example in [1], where the author uses the Baire Category Theorem to show that for a continuous $n$-dimensional manifold $M$ with boundary, a typical element of the space of deformations of the boundary is nowhere-differentiable with Hausdorff dimension $n-1$.

One may ask whether star bodies with a fractal boundary exist, so we begin by introducing various examples of constructions that give us fractal star bodies, and we describe several operations on fractal star bodies, such as the star dual and suspension.

This paper shows also how the concepts from the two distinct streams of geometry interact for fractal star bodies. An important concept of convex geometry is the kernel of a star body. We show that the dimension of the kernel of a star body in $\mathbb{R}^{n}$ with fractal boundary must be less than or equal to $n-1$ or $n-2$ depending on the chosen fractal dimension.

This paper is only an introduction to the topic of fractal star bodies to obtain an idea of the questions surrounding them. There are some interesting open questions related to the idea of self-similarity and how this could be used to create fractal star bodies 
with a boundary of a prescribed dimension. Although the boundary of a star body could never be strictly self-similar, there are notions of generalized self-similarity that would be applicable to fractal star bodies. We will discuss these in forthcoming research.

Section 1 gives an axiomatic definition of fractal dimension and explains what we mean by a fractal set.

In Section 2 we define the notion of fractal star body and discuss some of its basic properties.

In Section 3 we introduce some families of star bodies that are fractal with respect to a given fractal dimension. We show how such objects can be constructed starting with real-valued functions defined on a subset of Euclidean space, for which the graph is a fractal set.

Sections 4 and 5 discuss various operations on the family of fractal star bodies.

In Section 6 we give an upper bound for the topological dimension of the kernel of a fractal star body.

1. Fractals and locally fractal sets. Since there is no generally accepted definition of fractal, we shall begin with terminology and notation to be used in this paper.

All the metric spaces considered are assumed to be separable and 'dim' will denote the topological dimension (see [4]). (In fact, for this paper, it is sufficient to consider only subspaces of $\mathbb{R}^{n}$.) In [5], p. 29, Falconer lists certain properties of the Hausdorff dimension (defined in Section 2.2, op. cit.), which he suggests should be taken as conditions for any reasonable definition of dimension.

Based on this, we define a fractal dimension to be any function $\operatorname{dim}_{F}$ which assigns to a nonempty metric space $(X, d)$ a non-negative real number $\operatorname{dim}_{F}(X, d)$, also written as $\operatorname{dim}_{F} X$ if this does not lead to confusion, such that the following axioms are satisfied:

F.0. $\operatorname{dim}_{F} X \geq \operatorname{dim} X$;

F.1. (Lipschitz Subinvariance) If $f: X \rightarrow Y$ is Lipschitz with respect to $d_{X}$ and $d_{Y}$, then

$$
\operatorname{dim}_{F}\left(f(X), d_{Y}\right) \leq \operatorname{dim}_{F}\left(X, d_{X}\right) ;
$$

F.2. (Finite Stability) If $X=X_{1} \cup X_{2}$, then

$$
\operatorname{dim}_{F} X=\max \left\{\operatorname{dim}_{F} X_{1}, \operatorname{dim}_{F} X_{2}\right\} ;
$$

F.3. If $X$ is a singleton or a relatively open $k$-dimensional Euclidean ball for some natural number $k$, then $\operatorname{dim}_{F} X=\operatorname{dim} X$.

The fractal dimension $\operatorname{dim}_{F}$ is called $\sigma$-stable whenever it satisfies the axiom

F.2'. ( $\sigma$-Stability) If $X=\bigcup_{i=1}^{\infty} X_{i}$, then

$$
\operatorname{dim}_{F} X=\sup _{i \in \mathbb{N}} \operatorname{dim}_{F} X_{i}
$$

We have added Axiom F.0 (compare with [10], p. 107), we have omitted monotonicity, since it is implied by the Finite Stability Axiom F.2, and we have replaced the conditions concerning the dimensions of open subsets of $\mathbb{R}^{n}$ and smooth manifolds by Axiom F.3. The Hausdorff dimension satisfies this new set of axioms (a proof for Axiom F.0 can be found 
in [10], p. 107). Aside from the Hausdorff dimension, the most commonly used fractal dimension is the Minkowski dimension $\operatorname{dim}_{M}$, also called the box dimension, and denoted by $\operatorname{dim}_{B}$ in [5]. There are various equivalent descriptions of the Minkowski dimension (see for example Section 3.1 of [5]), some of which are only applicable to subspaces of $\mathbb{R}^{n}, n$-dimensional Euclidean space. The Hausdorff dimension is $\sigma$-stable, whereas the box dimension is only finitely stable, and $\operatorname{dim}_{M} \geq \operatorname{dim}_{H}$ (see [5]). The fact that $\operatorname{dim}_{M}$ satisfies these axioms, is mentioned on page 44 of [5].

For self-similar fractals which satisfy the open set condition these two dimensions coincide. This is in particular true for many IFS-fractals. Moreover, for such self-similar fractals these dimensions also agree with the similarity dimension as introduced on p. 64 and 65 of [12]. This provides us with a quick way to calculate the fractal dimension of such fractals. However, note that although the similarity dimension is most easily understood and therefore also most well-advertised, this dimension does not satisfy the conditions above (in particular, Axiom F.2) for arbitrary self-similar sets.

Definition 1.1. Let $A$ be a subset of $(X, d)$.

(i) The set $A$ is a fractal provided that for some fractal dimension $\operatorname{dim}_{F}$

$$
\operatorname{dim}_{F} A>\operatorname{dim} A .
$$

(ii) The set $A$ is locally fractal at $x \in A$ if for every neighbourhood $U$ of $x$ in $A$ there exists a neighbourhood $U_{0} \subseteq U$ of $x$ in $A$ such that $U_{0}$ is a fractal.

(iii) The set $A$ is locally fractal if $A$ is locally fractal at $x$ for every $x \in A$.

The reader will recognize the first part of this definition as one of the common ways to define the notion of fractal. However, if one also likes to think of fractals as spaces with arbitrary fine structure at all levels, this does not fully capture that, as one might take a union of a fractal with a space which is not fractal, but of a lower dimension. This will still be a fractal by this definition. To distinguish this from spaces for which every open subset is fractal, we introduce the notion of being locally fractal (where the word "locally" is used as in "locally connected").

Sometimes it is convenient to say (in the obvious sense) that $A$ is a fractal, locally fractal at $x$, or locally fractal, with respect to $\operatorname{dim}_{F}$.

Proposition 1.2. If $A$ is a compact fractal, there exists a point $x \in A$ such that $A$ is locally fractal at $x$.

Proof. We will prove the following slightly stronger statement: Let $\operatorname{dim}_{F}$ be a fractal dimension. For every compact space $A$ which is fractal with respect to $\operatorname{dim}_{F}$ there is a point $x \in A$, such that $A$ is locally fractal at $x$ with respect to $\operatorname{dim}_{F}$.

So let $A$ be a compact fractal with respect to $\operatorname{dim}_{F}$, and suppose, to the contrary, that for every $x \in A$ there is a neighbourhood $U_{x}$ of $x$ such that there is no neighbourhood $V_{x} \subseteq U_{x}$ of $x$ which is fractal with respect to $\operatorname{dim}_{F}$. Since $A$ is compact, this implies that $A$ is a finite union of subsets $U_{x_{1}}, \ldots, U_{x_{k}}$ with $\operatorname{dim}_{F} U_{x_{i}} \leq \operatorname{dim} U_{x_{i}}$ for $i=1, \ldots, k$.

Thus, by Axiom F.2, $\operatorname{dim}_{F} A \leq \operatorname{dim} A$ for every $\operatorname{dim}_{F}$. This contradicts the fact that $A$ is fractal with respect to $\operatorname{dim}_{F}$. 
The converse of this proposition is not true since one may have $A=A_{0} \cup A_{1}$ with $A_{0}$ locally fractal and $\operatorname{dim}_{F} A_{1}=\operatorname{dim} A_{1} \geq \operatorname{dim}_{F} A_{0}$, so that $\operatorname{dim}_{F} A=\operatorname{dim} A$. However, the following result is evident.

Proposition 1.3. Every compact set which is locally fractal is a fractal.

It is obvious that generally the converse implication does not hold. However, it is easy to verify the following.

EXAMPLE 1.4. Every fractal in $\mathbb{R}^{n}$ which is the attractor of some set of contractions is a local fractal.

By Axiom F.1 and Definition 1.1, we obtain

Proposition 1.5. Both the notion of fractal set and that of locally fractal set are invariant under bilipschitz maps.

2. Fractal star bodies and their basic properties. Let $a_{0}, a_{1}, \ldots, a_{k}$ be points in $\mathbb{R}^{n}$. In what follows we shall use the symbol $\Delta\left(a_{0}, \ldots, a_{k}\right)$ to denote the $k$-simplex in $\mathbb{R}^{n}$ with vertices $a_{0}, \ldots, a_{k}$; in particular, $\Delta\left(a_{0}, a_{1}\right)$ is the segment with endpoints $a_{0}$, and $a_{1}$, where $a_{0} \neq a_{1}$. Note that the $k$-simplex is $k$-dimensional when the points $a_{0}, \ldots, a_{k}$ are affinely independent. When this is not the case, we call it degenerate.

We shall refer to a subset $A$ of $\mathbb{R}^{n}$ as a star body if $A$ is compact, $A=\operatorname{cl}$ int $A$ and there is an $a \in A$ such that the segment $\Delta(a, x)$ is contained in $A$ whenever $x \in A$; that is, intuitively, every point $x$ of $A$ is visible from $a$. The kernel of $A$ is the set $\operatorname{ker} A$ of all points of $A$ from which every point of $A$ is "visible".

For any star body $A$ in $\mathbb{R}^{n}$ with $0 \in \operatorname{ker} A$, let $\rho_{A}: S^{n-1} \rightarrow \mathbb{R}_{+}$be the radial function of $A$ :

$$
\rho_{A}(u):=\sup \{\lambda \geq 0 \mid \lambda u \in A\} .
$$

We shall consider two families $\mathcal{S}^{n}$ and $\tilde{\mathcal{S}}^{n}$ defined as follows:

Definition 2.1. A star body $A$ in $\mathbb{R}^{n}$ belongs to the family $\mathcal{S}^{n}$ if and only if $0 \in \operatorname{ker} A$ and $\rho_{A}$ is positive and continuous. Furthermore, $A \in \tilde{\mathcal{S}}^{n}$ if and only if $0 \in \operatorname{ker} A$ and $\rho_{A}$ is continuous.

It is easy to see that

- if $A \in \mathcal{S}^{n}$ then 0 does not separate $A$, because $0 \in \operatorname{int} A$;

- if $A \in \tilde{\mathcal{S}}^{n}$, then 0 may separate $A$ and, if it does, then for each connected component $C$ of $A \backslash\{0\}$, the star body $C \cup\{0\}$ (that is, the closure of $C$ ) belongs again to $\tilde{\mathcal{S}}^{n}$. An easy example is the star body $A:=\left(a+B^{n}\right) \cup\left(-a+B^{n}\right)$, the union of two translates of the unit ball $B^{n}$, for any $a \in \mathbb{R}^{n}$ with $\|a\|=1$.

We say that two metrics $d^{\prime}$ and $d^{\prime \prime}$ on a set $X$ are geometrically equivalent if $\mathrm{id}_{X}$ is a bilipschitz map from $\left(X, d^{\prime}\right)$ to $\left(X, d^{\prime \prime}\right)$. In particular, this means that $\operatorname{dim}_{F}\left(X, d^{\prime}\right)=$ $\operatorname{dim}_{F}\left(X, d^{\prime \prime}\right)$, for all fractal dimensions $\operatorname{dim}_{F}$. For fractal star bodies the metrics in the following lemma will be relevant. 
Lemma 2.2. For arbitrary metric spaces $\left(X_{i}, d_{i}\right), i=1,2$, the following three metrics on $X_{1} \times X_{2}$ are geometrically equivalent: for $x=\left(x_{1}, x_{2}\right)$ and $y=\left(y_{1}, y_{2}\right)$,

$$
\begin{aligned}
d(x, y) & :=\sqrt{\left(d_{1}\left(x_{1}, y_{1}\right)\right)^{2}+\left(d_{2}\left(x_{2}, y_{2}\right)\right)^{2}} ; \\
\bar{d}(x, y) & :=d_{1}\left(x_{1}, y_{1}\right)+d_{2}\left(x_{2}, y_{2}\right) ; \\
d_{\max }(x, y) & :=\max \left\{d_{1}\left(x_{1}, y_{1}\right), d_{2}\left(x_{2}, y_{2}\right)\right\} .
\end{aligned}
$$

Proof. It is easy to see that

$$
d \leq d_{1}+d_{2} \leq 2 d \text { and } d_{\max } \leq d_{1}+d_{2} \leq 2 d_{\max }
$$

Theorem 2.3. For every $A \in \mathcal{S}^{n}$, the function $f: \operatorname{graph}\left(\rho_{A}\right) \rightarrow \mathrm{bd} A$ defined by

$$
f\left(u, \rho_{A}(u)\right)=u \rho_{A}(u)
$$

is bilipschitz.

Proof. Let

$$
\alpha:=\max \left\{1, \sup \rho_{A}\right\} \quad \text { and } \quad \beta:=\min \left\{1, \inf \rho_{A}\right\} .
$$

It is clear that $\alpha \geq 1>0$, and the condition that $A \in \mathcal{S}^{n}$ implies that $\beta>0$.

Note that graph $\left(\rho_{A}\right) \subseteq S^{n-1} \times \mathbb{R}_{+}$, and its metric is the product metric induced by the Euclidean metrics on $S^{n-1}$ and $\mathbb{R}_{+}$. (Note that we take $S^{n-1}$ with the subspace metric, not with the intrinsic metric, which would have been the spherical metric.) By Lemma 2.2 , we may replace this product metric $d$ on $S^{n-1} \times \mathbb{R}_{+}$by $\bar{d}$ or by $d_{\max }$. So it suffices to show that

$$
d\left(f\left(u, \rho_{A}(u)\right), f\left(v, \rho_{A}(v)\right)\right) \leq \alpha \bar{d}\left(\left(u, \rho_{A}(u)\right),\left(v, \rho_{A}(v)\right)\right)
$$

and

$$
d\left(f\left(u, \rho_{A}(u)\right), f\left(v, \rho_{A}(v)\right)\right) \geq \beta \max \left\{\|u-v\|,\left|\rho_{A}(u)-\rho_{A}(v)\right|\right\} .
$$

Evidently,

$$
\begin{aligned}
d\left(f\left(u, \rho_{A}(u)\right), f\left(v, \rho_{A}(v)\right)\right) & =\left\|u \rho_{A}(u)-v \rho_{A}(v)\right\| \\
& =\left\|u\left(\rho_{A}(u)-\rho_{A}(v)\right)+(u-v) \rho_{A}(v)\right\| \\
& \leq\left|\rho_{A}(u)-\rho_{A}(v)\right|+\|u-v\| \rho_{A}(v) \\
& \leq \alpha \bar{d}\left(\left(u, \rho_{A}(u)\right),\left(v, \rho_{A}(v)\right)\right) ;
\end{aligned}
$$

thus, (2) holds.

To prove (3), without loss of generality we may assume that $\rho_{A}(u) \leq \rho_{A}(v)$. Let us consider the triangle $\Delta\left(0, u \rho_{A}(u), v \rho_{A}(u)\right.$ ) (which is degenerate when $u= \pm v$ ). Note that its external angle at the vertex $v \rho_{A}(u)$ is greater than or equal to $\frac{\pi}{2}$. Therefore,

$$
\begin{aligned}
d\left(f\left(u, \rho_{A}(u)\right), f\left(v, \rho_{A}(v)\right)\right) & \geq \max \left\{\left|\rho_{A}(u)-\rho_{A}(v)\right|,\|u-v\| \rho_{A}(u)\right\} \\
& \geq \beta \max \left\{\|u-v\|,\left|\rho_{A}(u)-\rho_{A}(v)\right|\right\} ;
\end{aligned}
$$

thus, (3) holds. We conclude that $f$ in (1) is bilipschitz.

REMARK 2.4. (a) The assumption $0 \in \operatorname{int} A$ (or, equivalently, $\rho_{A}$ is positive) is essential in Theorem 2.3. Indeed, let $A$ be the union of two discs $B_{1}, B_{2}$ in $\mathbb{R}^{2}$ with $B_{1} \cap B_{2}=\{0\}$. Then $A$ is a star body with $0 \in \operatorname{ker} A$ and $\rho_{A}$ is continuous, but bd $A$ is the union of two circles while graph $\rho_{A}$ is homeomorphic to a circle. 
(b) The result of Theorem 2.3 does apply to $A \in \tilde{\mathcal{S}}^{n}$ with the restricted function $f: \operatorname{graph}\left(\rho_{A} \mid C\right) \rightarrow\left\{u \cdot \rho_{A}(u) \mid u \in C\right\}$, for any nonempty compact subset $C$ of $S^{n-1} \backslash$ $\rho_{A}^{-1}(0)$.

Corollary 2.5. (i) If $A \in \mathcal{S}^{n}$, then

$$
\operatorname{dim}_{F} \operatorname{graph}\left(\rho_{A}\right)=\operatorname{dim}_{F} \operatorname{bd} A
$$

for every fractal dimension $\operatorname{dim}_{F}$.

(ii) If $A \in \tilde{\mathcal{S}}^{n}$ and $\operatorname{dim}_{F}$ is $\sigma$-stable, then again

$$
\operatorname{dim}_{F} \operatorname{graph}\left(\rho_{A}\right)=\operatorname{dim}_{F} \text { bd } A .
$$

Proof. Part (i) is a direct consequence of Theorem 2.3 combined with Axiom F.1.

For Part (ii), note that since $(0, \infty)=\bigcup_{k=1}^{\infty}[1 / k, \infty)$, it follows that

$$
S^{n-1} \backslash \rho_{A}^{-1}(0)=\bigcup_{k=1}^{\infty} \rho_{A}^{-1}[1 / k, \infty) .
$$

Let $C_{k}:=\rho_{A}^{-1}[1 / k, \infty)$ for every $k$. Since $\rho_{A}$ is continuous, each $C_{k}$ is compact and thus by Remark 2.4 (b), the map $\left(u, \rho_{A}(u)\right) \mapsto u \cdot \rho_{A}(u)$ for $u \in C_{k}$ is bilipschitz, whence, by Axioms F.2' and F.1,

$$
\begin{aligned}
\operatorname{dim}_{F}\left\{\left(u, \rho_{A}(u)\right) \mid \rho_{A}(u) \neq 0\right\} & =\operatorname{dim}_{F} \operatorname{graph}\left(\rho_{A} \mid \bigcup_{k=1}^{n} C_{k}\right)=\sup _{k \in \mathbb{N}} \operatorname{dim}_{F} \operatorname{graph}\left(\rho_{A} \mid C_{k}\right) \\
& =\operatorname{dim}_{F}(\operatorname{bd} A \backslash\{0\}) .
\end{aligned}
$$

Further, the remaining part of graph $\rho_{A}$ is a subset of $S^{n-1}$. So Axiom F.3 with Axiom F.1 implies that its fractal dimension is less than or equal to $n-1$; thus,

$$
\operatorname{dim}_{F} \operatorname{graph} \rho_{A}=\max \left\{n-1, \operatorname{dim}_{F}(\operatorname{bd} A \backslash\{0\})\right\} .
$$

Let us now look at the right hand side of the required equality (4). By Axiom F.0,

$$
\operatorname{dim}_{F} \operatorname{bd} A \geq \operatorname{dim} \operatorname{bd} A,
$$

while $\operatorname{dim} \operatorname{bd} A=n-1$ (see Corollary 2 on p. 46 in [10]), because $A$ is a body (that is, $A=\operatorname{clint} A$ ).

Finally, by Axiom F.2,

$$
\operatorname{dim}_{F} \operatorname{bd} A=\operatorname{dim}_{F}(\operatorname{bd} A \backslash\{0\}),
$$

because bd $A=\{0\} \cup(\operatorname{bd} A \backslash\{0\})$.

Definition 2.6. Let $A$ be a star body.

(i) $A$ is a fractal star body (with respect to $\operatorname{dim}_{F}$ ) if and only if bd $A$ is a fractal (with respect to $\operatorname{dim}_{F}$ );

(ii) $A$ is a locally fractal star body (with respect to $\operatorname{dim}_{F}$ ) if and only if bd $A$ is locally fractal (with respect to $\operatorname{dim}_{F}$ ).

Since every bilipschitz map is a homeomorphism and thus preserves the topological dimension, another direct consequence of Theorem 2.3 is the following. 
Corollary 2.7. For any $A \in \mathcal{S}^{n}$ and any fractal dimension $\operatorname{dim}_{F}, A$ is a fractal star body with respect to $\operatorname{dim}_{F}$ if and only if graph $\rho_{A}$ is fractal with respect to $\operatorname{dim}_{F}$. The same holds if $A \in \tilde{\mathcal{S}}^{n}$ and $\operatorname{dim}_{F}$ is $\sigma$-stable.

3. Constructions of examples of fractal star bodies. We are interested in constructions of examples of fractal star bodies and even more, of locally fractal star bodies. Our first starting point for such constructions is the set of continuous real-valued functions on $\mathbb{R}^{n-1}$ whose graphs are fractal or locally fractal (see, for instance, [11]). The additional assumption we require is that the restriction of such a function $\phi: \mathbb{R}^{n-1} \rightarrow \mathbb{R}$ to the boundary of some cube is a positive constant.

In what follows, $\left(e_{1}, \ldots, e_{n}\right)$ is the canonical basis of $\mathbb{R}^{n}$.

EXAmple 3.1. Let $P$ be a cube in $\mathbb{R}^{n-1}$ with facets parallel to the coordinate hyperplanes and with symmetry center 0 . Let $\operatorname{diam} P<1$.

Let $\phi: P \rightarrow \mathbb{R}$ be a continuous positive function with $\phi(x)=1$ for every $x \in$ bd $P$ such that graph $\phi$ is a fractal.

Take the upper half sphere $S$ of $S^{n-1}$; let $\pi$ be its orthogonal projection onto the hyperplane $\operatorname{lin}\left(e_{1}, \ldots, e_{n-1}\right)$ and let $S_{0}:=\pi^{-1}(P)$.

It is easy to show that the restriction map $\pi \mid S_{0}$ is bilipschitz. Hence, the product map $\left(\pi \mid S_{0}\right) \times \operatorname{id}_{\mathbb{R}_{+}}: S_{0} \times \mathbb{R}_{+} \rightarrow P \times \mathbb{R}_{+}$is bilipschitz too, and so is its restriction to graph $\phi \subseteq S_{0} \times \mathbb{R}_{+}$. Since, by the assumption, graph $\phi$ is fractal with respect to some $\operatorname{dim}_{F}$, so is its inverse image. This inverse image is the graph of the function $\phi \circ \pi$ on $S_{0}$, which is equal to 1 on the boundary of $S$. Its extension by 1 on $S^{n-1}$ is positive and continuous and thus is a radial function of some star body. By Theorem 2.3 and Corollary 2.4, this is a fractal star body with respect to $\operatorname{dim}_{F}$. Obviously, this is not a locally fractal star body.

Another way in which we can use functions with fractal graphs to construct fractal star bodies is through a parametrization of the boundary of a star body by spherical coordinates. For $n \geq 2$, let $X_{n}$ and $p_{n}: X_{n} \rightarrow S^{n-1}$ be defined as follows. For $n=2$ :

$$
X_{2}:=[0,2 \pi], \quad p_{2}\left(t_{1}\right):=\left(\cos t_{1}, \sin t_{1}\right) .
$$

If $n \geq 3$, then

$$
X_{n}:=[0, \pi]^{n-2} \times[0,2 \pi]=[0, \pi] \times X_{n-1} .
$$

To describe $p_{n}$, we identify $S^{n-1} \cap\left(e_{1}\right)^{\perp}$ with $S^{n-2}$ and, given $p_{n-1}: X_{n-1} \rightarrow S^{n-2}$, we define $p_{n}: X_{n} \rightarrow S^{n-1}$ by

$$
p_{n}\left(t_{1}, \ldots, t_{n-1}\right):=\left(\cos t_{1}\right) e_{1}+\left(\sin t_{1}\right) p_{n-1}\left(t_{2}, \ldots, t_{n-1}\right) .
$$

Note that

$$
p_{n}\left(0, t_{2}, \ldots, t_{n-1}\right)=e_{1} \quad \text { and } \quad p_{n}\left(\pi, t_{2}, \ldots, t_{n-1}\right)=-e_{1}, \text { for any } t_{2}, \ldots, t_{n-1} .
$$

Moreover,

$$
p_{n}\left(t_{1}, \ldots, t_{n-2}, 0\right)=p_{n}\left(t_{1}, \ldots, t_{n-2}, 2 \pi\right), \quad \text { for any } t_{1}, \ldots, t_{n-2} .
$$

So $p_{n}$ is obviously not bilipschitz on its domain $X_{n}$. However, we will show that there is a dense subset of this domain which can be covered by a countable collection of subsets on 
which $p_{n}$ is bilipschitz. As a consequence of this we will find that for the radial function $\rho_{A}: S^{n-1} \rightarrow \mathbb{R}_{+}$of a star body, composition with $p_{n}$ does not change the fractal dimension of its graph, i.e., that $\operatorname{dim}_{F}\left(\operatorname{graph} \rho_{A}\right)=\operatorname{dim}_{F}\left(\operatorname{graph} \rho_{A} \circ p_{n}\right)$ if $\operatorname{dim}_{F}$ is $\sigma$-stable. This is a useful result as it is often easier to find the fractal dimension of graph $\rho_{A} \circ p_{n}$ than that of graph $\rho_{A}$.

For $n \geq 2$, we will consider the following family of subsets of $X_{n}$. For any $k=$ $\left(k_{1}, \ldots, k_{n-1}\right) \in\{0,1\}^{n-1}$ and $\varepsilon \in(0, \pi / 4)$, the subset $X_{n}(k, \varepsilon) \subset X_{n}$ is defined by

$$
\begin{aligned}
& X_{2}(k, \varepsilon)=\left[k_{1} \pi+\varepsilon,\left(k_{1}+1\right) \pi-\varepsilon\right] ; \\
& X_{n}(k, \varepsilon)=\prod_{i=1}^{n-2}\left[k_{i} \frac{\pi}{2}+\varepsilon,\left(k_{i}+1\right) \frac{\pi}{2}-\varepsilon\right] \times\left[k_{n-1} \pi+\varepsilon,\left(k_{n-1}+1\right) \pi-\varepsilon\right] .
\end{aligned}
$$

Note that the subsets $X_{n}(k, \varepsilon)$ can be defined inductively as

$$
\begin{aligned}
& X_{2}(k, \varepsilon)=\left[k_{1} \pi+\varepsilon,\left(k_{1}+1\right) \pi-\varepsilon\right] \\
& X_{n}(k, \varepsilon)=\left[k_{1} \frac{\pi}{2}+\varepsilon,\left(k_{1}+1\right) \frac{\pi}{2}-\varepsilon\right] \times X_{n-1}(\bar{k}, \varepsilon),
\end{aligned}
$$

where $\bar{k}=\left(k_{2}, \ldots, k_{n-1}\right)$ and $n \geq 3$.

Lemma 3.2. Let $n \geq 2$. For every $k=\left(k_{1}, \ldots, k_{n-1}\right) \in\{0,1\}^{n-1}$ and $\varepsilon \in\left(0, \frac{\pi}{4}\right)$, the restricted function $p_{n} \mid X_{n}(k, \varepsilon): X_{n}(k, \varepsilon) \rightarrow p_{n}\left(X_{n}(k, \varepsilon)\right)$ is bilipschitz.

Proof. Let $k \in\{0,1\}^{n-1}$ and $\varepsilon \in\left(0, \frac{\pi}{4}\right)$ be given. We need to prove that there are positive $\alpha_{n}$ and $\beta_{n}$ such that for all $t, t^{\prime} \in X_{n}(k, \varepsilon)$,

$$
\beta_{n}\left\|t-t^{\prime}\right\| \leq\left\|p_{n}(t)-p_{n}\left(t^{\prime}\right)\right\| \leq \alpha_{n}\left\|t-t^{\prime}\right\| .
$$

For $p_{2}\left(t_{1}\right)=\left(\cos t_{1}, \sin t_{1}\right)($ as in $(5))$, we calculate

$$
\begin{aligned}
\left\|p_{2}\left(t_{1}\right)-p_{2}\left(t_{1}^{\prime}\right)\right\| & =\sqrt{\left(\cos t_{1}-\cos t_{1}^{\prime}\right)^{2}+\left(\sin t_{1}-\sin t_{1}^{\prime}\right)^{2}} \\
& \leq \sqrt{\left(t_{1}-t_{1}^{\prime}\right)^{2}+\left(t_{1}-t_{1}^{\prime}\right)^{2}}=\sqrt{2}\left|t_{1}-t_{1}^{\prime}\right|,
\end{aligned}
$$

where the inequality is obtained by observing that $\left|\cos ^{\prime}(t)\right| \leq 1$ and $\left|\sin ^{\prime}(t)\right| \leq 1$. So we may take $\alpha_{2}=\sqrt{2}$.

On the other hand,

$$
\begin{aligned}
\left\|p_{2}\left(t_{1}\right)-p_{2}\left(t_{1}^{\prime}\right)\right\| & \geq \frac{1}{2} \max \left\{\left|\cos t_{1}-\cos t_{1}^{\prime}\right|,\left|\sin t_{1}-\sin t_{1}^{\prime}\right|\right\} \\
& \geq \frac{1}{2} \min _{s \in\left[k_{1} \pi,\left(k_{1}+1\right) \pi\right]} \max \left\{\left|\cos ^{\prime} s\right|,\left|\sin ^{\prime} s\right|\right\}\left|t_{1}-t_{1}^{\prime}\right| \geq \frac{\sqrt{2}}{4}\left|t_{1}-t_{1}^{\prime}\right|,
\end{aligned}
$$

for both values of $k_{1}$. So we may take $\beta_{2}=\frac{\sqrt{2}}{4}$. This proves the assertion for $n=2$.

By induction, assume that $p_{n-1}$ is bilipschitz on $X_{n-1}(\bar{k}, \varepsilon)$ (for $n \geq 3$ and $\bar{k}=$ $\left.\left(k_{2}, \ldots, k_{n-1}\right)\right)$.

For the induction step, we need to prove the existence of positive constants $\alpha_{n}$ and $\beta_{n}$ such that for $t=\left(t_{1}, \ldots, t_{n-1}\right)$ and $t^{\prime}=\left(t_{1}^{\prime}, \ldots, t_{n-1}^{\prime}\right)$ in $X_{n}(k, \varepsilon)$,

$$
\beta_{n}\left\|t-t^{\prime}\right\| \leq\left\|p_{n}(t)-p_{n}\left(t^{\prime}\right)\right\| \leq \alpha_{n}\left\|t-t^{\prime}\right\| .
$$

Let $\bar{t}=\left(t_{2}, \ldots, t_{n-1}\right)$ and $\bar{t}^{\prime}=\left(t_{2}^{\prime}, \ldots, t_{n-1}^{\prime}\right)$. 
By the inductive definition of $p_{n}$ in $(7)$,

$$
p_{n}(t)-p_{n}\left(t^{\prime}\right)=\left(\cos t_{1}-\cos t_{1}^{\prime}\right) e_{1}+\sin t_{1} p_{n-1}(\bar{t})-\sin t_{1}^{\prime} p_{n-1}\left(\bar{t}^{\prime}\right) .
$$

We will first find the constant $\alpha_{n}$ for the upper bound. By Lemma 2.2 we may replace $\left\|p_{n}(t)-p_{n}\left(t^{\prime}\right)\right\|$ by $\left|\cos t_{1}-\cos t_{1}^{\prime}\right|+\left\|\sin t_{1} p_{n-1}(\bar{t})-\sin t_{1}^{\prime} p_{n-1}\left(\bar{t}^{\prime}\right)\right\|$. Observe that

$$
\begin{aligned}
& \left\|\sin t_{1} p_{n-1}(\bar{t})-\sin t_{1}^{\prime} p_{n-1}\left(\bar{t}^{\prime}\right)\right\| \\
& =\left\|\left(\sin t_{1}-\sin t_{1}^{\prime}\right) p_{n-1}(\bar{t})+\left(\sin t_{1}^{\prime}\right)\left(p_{n-1}(\bar{t})-p_{n-1}\left(\bar{t}^{\prime}\right)\right)\right\| \\
& \leq\left|\sin t_{1}-\sin t_{1}^{\prime}\right|+\left\|p_{n-1}(\bar{t})-p_{n-1}\left(\bar{t}^{\prime}\right)\right\|,
\end{aligned}
$$

because $p_{n-1}(\bar{t}) \in S^{n-2}$.

Since $\cos$ and $\sin$ are Lipschitz on $\left[k_{1} \frac{\pi}{2}+\varepsilon,\left(k_{1}+1\right) \frac{\pi}{2}-\varepsilon\right]$, there are positive constants $\lambda$ and $\mu$ such that $\left|\cos t_{1}-\cos t_{1}^{\prime}\right| \leq \lambda\left|t_{1}-t_{1}^{\prime}\right|$ and $\left|\sin t_{1}-\sin t_{1}^{\prime}\right| \leq \mu\left|t_{1}-t_{1}^{\prime}\right|$.

We conclude that

$$
\left|\cos t_{1}-\cos t_{1}^{\prime}\right|+\left\|\sin t_{1} p_{n-1}(\bar{t})-\sin t_{1}^{\prime} p_{n-1}\left(\bar{t}^{\prime}\right)\right\| \leq(\lambda+\mu)\left|t_{1}-t_{1}^{\prime}\right|+\alpha_{n-1}\left\|\bar{t}-\bar{t}^{\prime}\right\| .
$$

So we obtain the required

$$
\left|\cos t_{1}-\cos t_{1}^{\prime}\right|+\left\|\sin t_{1} p_{n-1}(\bar{t})-\sin t_{1}^{\prime} p_{n-1}\left(\bar{t}^{\prime}\right)\right\| \leq \alpha_{n}\left(\left|t_{1}-t_{1}^{\prime}\right|+\left\|\bar{t}-\bar{t}^{\prime}\right\|\right),
$$

by taking $\alpha_{n}=\max \left\{\lambda+\mu, \alpha_{n-1}\right\}$.

To find the constant $\beta_{n}$ for the lower bound, take $t, t^{\prime} \in X_{n}(k, \varepsilon)$. By possibly interchanging $t$ and $t^{\prime}$, we may assume that if $k_{1}=0$, then $t_{1} \leq t_{1}^{\prime}$ and if $k_{1}=1$, then $t_{1} \geq t_{1}^{\prime}$, so that

$$
\sin t_{1}-\sin t_{1}^{\prime} \leq 0
$$

Since

$$
\begin{aligned}
& p_{n}(t)-p_{n}\left(t^{\prime}\right) \\
& =\left(\cos t_{1}-\cos t_{1}^{\prime}\right) e_{1}+\left(\left(\sin t_{1}\right) p_{n-1}(\bar{t})-\left(\sin t_{1}^{\prime}\right) p_{n-1}\left(\bar{t}^{\prime}\right)\right) \\
& =\left(\cos t_{1}-\cos t_{1}^{\prime}\right) e_{1}+\left(\sin t_{1}\right)\left(p_{n-1}(\bar{t})-p_{n-1}\left(\bar{t}^{\prime}\right)\right)+p_{n-1}\left(\bar{t}^{\prime}\right)\left(\sin t_{1}-\sin t_{1}^{\prime}\right),
\end{aligned}
$$

it follows that

$$
\begin{aligned}
\left\|p_{n}(t)-p_{n}\left(t^{\prime}\right)\right\|^{2}= & \left.\left(\cos t_{1}-\cos t_{2}\right)^{2}+\sin ^{2} t_{1} \| p_{n-1}(\bar{t})-p_{n-1}\left(\bar{t}^{\prime}\right)\right) \|^{2}+ \\
& +\left(\sin t_{1}-\sin t_{1}^{\prime}\right)^{2}+2 \sin t_{1}\left(\sin t_{1}-\sin t_{1}^{\prime}\right)(\cos \phi-1),
\end{aligned}
$$

where $\phi:=\angle\left(p_{n-1}(\bar{t}), p_{n-1}\left(\bar{t}^{\prime}\right)\right)$.

Thus, by $(10)$,

$$
\left\|p_{n}(t)-p_{n}\left(t^{\prime}\right)\right\|^{2} \geq\left(\cos t_{1}-\cos t_{2}\right)^{2}+\left(\sin t_{1}-\sin t_{1}^{\prime}\right)^{2}+\left(\sin ^{2} \varepsilon\right)\left\|p_{n-1}(\bar{t})-p_{n-1}\left(\bar{t}^{\prime}\right)\right\|^{2},
$$

because the omitted term $2 \sin t_{1}\left(\sin t_{1}-\sin t_{1}^{\prime}\right)(\cos \phi-1)$ is non-negative and $\left|\sin t_{1}\right| \geq$ $|\sin \varepsilon|$ for $t_{1} \in\left[k_{1} \frac{\pi}{2}+\varepsilon,\left(k_{1}+1\right) \frac{\pi}{2}-\varepsilon\right]$.

Since

$$
\left(\cos t_{1}-\cos t_{1}^{\prime}\right)^{2}+\left(\sin t_{1}-\sin t_{1}^{\prime}\right)^{2}=\left\|p_{2}\left(t_{1}\right)-p_{2}\left(t_{1}^{\prime}\right)\right\|^{2} \geq \frac{1}{8}\left|t_{1}-t_{1}^{\prime}\right|^{2},
$$

as shown earlier in this proof, and

$$
\left\|p_{n-1}(\bar{t})-p_{n-1}\left(\bar{t}^{\prime}\right)\right\|^{2} \geq\left(\beta_{n-1}\right)^{2}\left\|\bar{t}-\bar{t}^{\prime}\right\|^{2},
$$


we obtain

$$
\left\|p_{n}(t)-p_{n}\left(t^{\prime}\right)\right\|^{2} \geq \frac{1}{8}\left|t_{1}-t_{1}^{\prime}\right|^{2}+\left((\sin \varepsilon) \beta_{n-1}\right)^{2}\left\|\bar{t}-\bar{t}^{\prime}\right\|^{2} .
$$

Thus, if

$$
\beta_{n}:=\sqrt{\min \left\{1 / 8,\left((\sin \varepsilon) \beta_{n-1}\right)^{2}\right\}}
$$

then

$$
\left\|p_{n}(t)-p_{n}\left(t^{\prime}\right)\right\| \geq \beta_{n} \sqrt{\left|t_{1}-t_{1}^{\prime}\right|^{2}+\left\|\bar{t}-\bar{t}^{\prime}\right\|^{2}}=\beta_{n}\left\|t-t^{\prime}\right\| .
$$

This concludes the proof of Lemma 3.2.

REMARK 3.3. It is easy to see that for $X(k):=X_{n}(k)$, defined by

$$
X_{2}\left(k_{1}\right)=\left[k_{1} \pi,\left(k_{1}+1\right) \pi\right] \text { and } X_{n}(k)=\left[k_{1} \frac{\pi}{2},\left(k_{1}+1\right) \frac{\pi}{2}\right] \times X_{n-1}(\bar{k}),
$$

and a continuous function $f: X(k) \rightarrow \mathbb{R}$,

$$
\operatorname{dim}_{F} \operatorname{graph}(f \mid \operatorname{int} X(k)) \geq \operatorname{dim}_{F} \operatorname{graph}(f \mid \operatorname{bd} X(k)),
$$

for arbitrary $\operatorname{dim}_{F}$.

Indeed, by Axiom F.0, the left hand side is greater than or equal to

$$
\operatorname{dim} \operatorname{graph}(f \mid \operatorname{int} X(k))=\operatorname{dimint} X(k)=n-1,
$$

while, by Axioms F.2 and F.3 together with Axiom F.1, the right hand side is less than or equal to $n-1$, because bd $X(k)$ is the union of the relative interiors of all the proper faces of bd $X(k)$, and so each part of this graph lies within a subspace of $\mathbb{R}^{n}$ which has dimension $n-1$.

Note that the above inequality and reasoning also applies when $X(k)$ is replaced by any other $(n-1)$-dimensional convex polytope in $\mathbb{R}^{n-1}$ or by a spherical polytope in $S^{n-1}$; for instance, it applies to $p(X(k))$.

TheOrem 3.4. Let $A \in \tilde{\mathcal{S}}^{n}$. If $X:=X_{n}$ and $p:=p_{n}$ are defined by (5)-(7) and

$$
r_{A}:=\rho_{A} \circ p
$$

then

$$
\operatorname{dim}_{F} \operatorname{graph} r_{A}=\operatorname{dim}_{F} \operatorname{graph} \rho_{A}
$$

for every $\sigma$-stable fractal dimension $\operatorname{dim}_{F}$.

Proof. Let $X(k)$ and $X(k, \varepsilon)$ be defined by (11) and (8) with (9), respectively. Note that for $k \in\{0,1\}^{n-1}$ and $\varepsilon \in\left(0, \frac{\pi}{4}\right)$,

$$
\operatorname{dim}_{F} \operatorname{graph}\left(r_{A} \mid X(k, \varepsilon)\right)=\operatorname{dim}_{F} \operatorname{graph}\left(\rho_{A} \mid p(X(k, \varepsilon))\right) .
$$

Indeed, by Lemma 3.2, the Cartesian product of $p \mid X(k, \varepsilon)$ and the identity on $r_{A}(X(k, \varepsilon))$ is bilipschitz. Using formula (12) we derive

$$
\begin{aligned}
\operatorname{dim}_{F} \operatorname{graph}\left(r_{A} \mid X(k, \varepsilon)\right) & =\operatorname{dim}_{F}\left\{\left(t, r_{A}(t)\right) \mid t \in X(k, \varepsilon)\right\} \\
& =\operatorname{dim}_{F}\left\{\left(p(t), r_{A}(t)\right) \mid t \in X(k, \varepsilon)\right\} \\
& =\operatorname{dim}_{F}\left\{\left(u, \rho_{A}(u)\right) \mid u \in p(X(k, \varepsilon))\right\} \\
& =\operatorname{dim}_{F} \operatorname{graph}\left(\rho_{A} \mid p(X(k, \varepsilon))\right) .
\end{aligned}
$$

This proves equality (13). 
Now take any sequence $\left(\varepsilon_{i}\right)_{i \in \mathbb{N}}$ which converges to 0 . Evidently,

$$
\operatorname{int} X(k)=\bigcup_{i \in \mathbb{N}} X\left(k, \varepsilon_{i}\right) \text {. }
$$

Thus, by Axiom F.2' ( $\sigma$-stability of $\left.\operatorname{dim}_{F}\right)$, combined with (13),

$$
\operatorname{dim}_{F} \operatorname{graph}\left(r_{A} \mid \operatorname{int} X(k)\right)=\operatorname{dim}_{F}\left(\operatorname{graph}\left(\rho_{A} \mid p(\operatorname{int} X(k))\right)\right) .
$$

Finally, by Remark 3.3,

$$
\operatorname{dim}_{F} \operatorname{graph}\left(r_{A} \mid \operatorname{int} X(k)\right) \geq \operatorname{dim}_{F} \operatorname{graph}\left(r_{A} \mid \operatorname{bd} X(k)\right),
$$

so we may conclude that

$$
\operatorname{dim}_{F} \operatorname{graph}\left(r_{A} \mid X(k)\right)=\operatorname{dim}_{F} \operatorname{graph}\left(r_{A} \mid \operatorname{int} X(k)\right) .
$$

Analogously,

$$
\begin{aligned}
\operatorname{dim}_{F}\left(\operatorname{graph}\left(\rho_{A} \mid p(\operatorname{int} X(k))\right)\right) & =\operatorname{dim}_{F}\left(\operatorname{graph}\left(\rho_{A} \mid \operatorname{int} p(X(k))\right)\right) \\
& =\operatorname{dim}_{F}\left(\operatorname{graph}\left(\rho_{A} \mid p(X(k))\right)\right) .
\end{aligned}
$$

By (14), this completes the proof.

Corollary 3.5. Let $A \in \tilde{\mathcal{S}}^{n}$ and let $\operatorname{dim}_{F}$ be $\sigma$-stable. If $r_{A}$ is defined as in (12) then the star body $A$ is fractal with respect to $\operatorname{dim}_{F}$ if and only if graph $r_{A}$ is fractal with respect to $\operatorname{dim}_{F}$.

Proposition 3.6. Let $n \geq 2$ and let $X_{n}$ and $p_{n}$ be defined by (5)-(7). For every function $r: X_{n} \rightarrow \mathbb{R}_{+}$the following are equivalent:

(i) ${ }_{n}$ there is a star body $A \in \tilde{\mathcal{S}}^{n}$ such that $r=\rho_{A} \circ p_{n}$;

$(\text { ii })_{n} r$ is continuous and satisfies the following conditions:

- if $n=2$, then $r(0)=r(2 \pi)$;

- if $n \geq 3$, then

* $r(t)=r\left(t^{\prime}\right)$ for every $t=\left(t_{1}, \ldots, t_{n-1}\right)$ and $t^{\prime}=\left(t_{1}^{\prime}, \ldots, t_{n-1}^{\prime}\right)$ with $t_{j}=t_{j}^{\prime}$ for $j \leq j_{0}$ and $t_{j_{0}}=0=t_{j_{0}}^{\prime}$ or $t_{j_{0}}=\pi=t_{j_{0}}^{\prime}$;

* $r\left(t_{1}, \ldots, t_{n-2}, 0\right)=r\left(t_{1}, \ldots, t_{n-2}, 2 \pi\right)$.

Proof. The equivalence $(\mathrm{i})_{2} \Leftrightarrow(\mathrm{ii})_{2}$ is evident.

Let $n \geq 3$. The implication (i) $)_{n} \Rightarrow(\text { ii })_{n}$ follows from the properties of $p_{n}$, as described after the definition in equation $(7)$ and the inductive nature of this definition.

To show that $(\mathrm{ii})_{n} \Rightarrow(\mathrm{i})_{n}$, let $X:=X_{n}$ and $p:=p_{n}$. Consider all the pairs of opposite facets $F_{i}, F_{i}^{\prime}$ of the polytope $X$ : for $i \leq n-2$,

$$
F_{i}:=\left\{t=\left(t_{1}, \ldots, t_{n-1}\right) \in X \mid t_{i}=0\right\}, \quad F_{i}^{\prime}:=\left\{t^{\prime}=\left(t_{1}^{\prime}, \ldots, t_{n-1}^{\prime}\right) \in X \mid t_{i}^{\prime}=\pi\right\} ;
$$

and

$$
F_{n-1}:=\left\{t=\left(t_{1}, \ldots, t_{n-2}, 0\right) \in X\right\}, \quad F_{n-1}^{\prime}:=\left\{t^{\prime}=\left(t_{1}^{\prime}, \ldots, t_{n-2}^{\prime}, 2 \pi\right) \in X\right\} .
$$

If $p_{2}\left(t_{1}\right)=p_{2}\left(t_{1}^{\prime}\right)$ and $t_{1} \neq t_{1}^{\prime}$, then $\left\{t_{1}, t_{1}^{\prime}\right\}=\{0,2 \pi\}$. For $n \geq 3$, if $p_{n}(t)=p_{n}\left(t^{\prime}\right)$ and $t \neq t^{\prime}$, then either $\sin \left(t_{1}\right)=0$ and $t_{1}=t_{1}^{\prime} \in\{0, \pi\}$, or $t_{1}=t_{1}^{\prime}$ and $p_{n-1}\left(t_{2}, \ldots, t_{n-1}\right)=$ $p_{n-1}\left(t_{2}^{\prime}, \ldots, t_{n-1}^{\prime}\right)$. It is easy to see by induction that $p$ identifies points on the facets of $X$; more precisely, it identifies $F_{n-1}$ with $F_{n-1}^{\prime}$ and it collapses parts of the other facets. 
Moreover, $p$ is a closed surjective map and it is not hard to see that $S^{n-1}$ has the quotient topology for $X$ with the equivalence relation induced by the pre-images of $p$.

So in order to show that $r: X \rightarrow \mathbb{R}_{+}$induces a continuous map $\rho: S^{n-1} \rightarrow \mathbb{R}_{+}$, we only need to show that it is well-defined, i.e., if $p(t)=p\left(t^{\prime}\right)$ then $r(t)=r\left(t^{\prime}\right)$. By (ii) ${ }_{n}$, this is indeed the case. Since $\rho$ is a continuous map with values in $\mathbb{R}_{+}$, it defines the radial function of a star body $A \in \tilde{\mathcal{S}}^{n}$. Thus (i) $n$ is satisfied. This completes the proof.

It is an open problem whether the condition that $\operatorname{dim}_{F}$ be $\sigma$-stable is essential in Corollary 3.5. However, the fact that the function $p_{n}: X_{n} \rightarrow S^{n-1}$ is Lipschitz on all of its domain implies that

$$
\operatorname{dim}_{F} \operatorname{graph}\left(\rho_{A} \circ p_{n}\right) \geq \operatorname{dim}_{F} \operatorname{graph}\left(\rho_{A}\right),
$$

for any fractal dimension (we don't need $\sigma$-stability for this inequality). So if the star body $A$ has a fractal boundary, we will also find that graph $\left(\rho_{A} \circ p_{n}\right)$ is fractal. More generally, we have the following result.

Proposition 3.7. There are rotations $\tau_{1}, \ldots, \tau_{n}$ of $\mathbb{R}^{n}$ such that for every $A \in \tilde{\mathcal{S}}^{n}$, the graph of $\rho_{A}$ is fractal with respect to $\operatorname{dim}_{F}$ if and only if there is an $i \in\{1, \ldots, n\}$, an $\varepsilon \in\left(0, \frac{\pi}{4}\right)$, and $a k \in\{0,1\}^{n-1}$ such that $\operatorname{graph}\left(\rho_{A} \circ \tau_{i} \circ p_{n} \mid X_{n}(k, \varepsilon)\right)$ is fractal with respect to $\operatorname{dim}_{F}$.

Proof. It is not hard to see that there are $n$ rotations $\tau_{1}, \ldots, \tau_{n}$ (including the identity) of $\mathbb{R}^{n}$ such that

$$
\bigcap_{i=1}^{n} \tau_{i} \circ p_{n}\left(\bigcup_{k \in\{0,1\}^{n-1}} \operatorname{bd} X_{n}(k)\right)=\emptyset .
$$

Let $\left\{\tau_{1}, \ldots, \tau_{n}\right\}$ be such a collection of $n$ rotations for the rest of this proof.

Suppose that graph $\left(\rho_{A}\right)$ is fractal with respect to $\operatorname{dim}_{F}$. Since graph $\left(\rho_{A}\right)$ is compact, there is a point $u \in S^{n-1}$ such that graph $\left(\rho_{A}\right)$ is locally fractal at $\left(u, \rho_{A}(u)\right)$ by Proposition 1.2. Since $\bigcap_{i=1}^{n} \tau_{i} \circ p_{n}\left(\bigcup_{k \in\{0,1\}^{n-1}}\right.$ bd $\left.X_{n}(k)\right)=\emptyset$, there is an $i \in\{1, \ldots, n\}$, a $k \in\{0,1\}^{n-1}$, and $\varepsilon>0$ such that $u \in \tau_{i} \circ p_{n}\left(X_{n}(k, \varepsilon)\right)$. The function $\tau_{i} \circ p_{n}$ is bilipschitz on $X_{n}(k, \varepsilon), \operatorname{so~} \operatorname{dim}_{F}\left(\operatorname{graph}\left(\rho_{A} \circ \tau_{i} \circ p_{n} \mid X_{n}(k, \varepsilon)\right)\right)=\operatorname{dim}_{F}\left(\operatorname{graph}\left(\rho_{A} \mid \tau_{i} \circ p_{n}\left(X_{n}(k, \varepsilon)\right)\right)\right)>$ $n-1$. We conclude that graph $\left(\rho_{A} \circ \tau_{i} \circ p_{n} \mid X_{n}(k, \varepsilon)\right)$ is fractal with respect to $\operatorname{dim}_{F}$.

Conversely, assume that graph $\left(\rho_{A} \circ \tau_{i} \circ p_{n} \mid X_{n}(k, \varepsilon)\right)$ is fractal with respect to $\operatorname{dim}_{F}$, for some values of $i, k$, and $\varepsilon$. Since $\tau_{i} \circ p_{n} \mid X_{n}(k, \varepsilon)$ is bilipschitz, it follows that graph $\left(\rho_{A} \mid \tau_{i} \circ\right.$ $\left.p_{n}\left(X_{n}(k, \varepsilon)\right)\right)$ is fractal with respect to $\operatorname{dim}_{F}$, so $\operatorname{dim}_{F}\left(\operatorname{graph}\left(\rho_{A} \mid \tau_{i} \circ p_{n}\left(X_{n}(k, \varepsilon)\right)\right)\right)>$ $n-1$. By Axiom F.2 this implies that $\operatorname{dim}_{F}\left(\operatorname{graph}\left(\rho_{A}\right)\right)>n-1, \operatorname{so} \operatorname{graph}\left(\rho_{A}\right)$ is fractal with respect to $\operatorname{dim}_{F}$.

EXAMPLE 3.8. Let $n=2$ and let $r:[0,2 \pi] \rightarrow \mathbb{R}$ be the Weierstrass function (see [5], p. 148, Example 11.3). For $\lambda \in(1, \infty)$ and $s \in(1,2)$, this function $r$ is defined by

$$
r(t)=\sum_{k=1}^{\infty} \lambda^{(s-2) k} \sin \lambda^{k} t .
$$

Then, for $\lambda$ large enough, $\operatorname{dim}_{M}$ graph $r=s$ and $\operatorname{dim}_{H} \operatorname{graph} r \geq s-c / \log \lambda$, for some constant $c$. So for $\lambda$ large enough, both $\operatorname{dim}_{M} \operatorname{graph} r>1$ and $\operatorname{dim}_{H} \operatorname{graph} r>1$. 
Defined this way, $r$ is a function with a fractal graph, both relative to $\operatorname{dim}_{H}$ and relative to $\operatorname{dim}_{M}$. However, we don't necessarily have that $r(0)=r(2 \pi)$, since $\lambda$ need not be an integer. We also need $r(t) \geq 0$ for all $t$. So we adjust the function as follows:

$$
\tilde{r}(t):=\mid r(t)+\left(r(2 \pi)+\frac{t}{2 \pi}(r(0)-r(2 \pi)) \mid+\gamma,\right.
$$

for some constant $\gamma \geq 0$. Then $\tilde{r}(0)=|r(0)+r(2 \pi)|+\gamma=\tilde{r}(2 \pi)$ and $\tilde{r}:[0,2 \pi] \rightarrow \mathbb{R}_{+}$ is continuous; thus, by Proposition 3.6 for $n=2$, there exists a star body $A \in \tilde{\mathcal{S}}^{2}$ such that $\tilde{r}(t)=\rho_{A}(\cos t, \sin t)$ for $t \in[0,2 \pi]$. Moreover, if $\gamma>0$, then $A \in \mathcal{S}^{2}$. Since the absolute value does not change the dimension of the graph of a function and the additional summand of $\tilde{r}$ is a Lipschitz function, Exercise 11.2 in [5] says that

$$
\operatorname{dim}_{F} \operatorname{graph} \tilde{r}=\max \left(1, \operatorname{dim}_{F} \operatorname{graph} r\right)=\operatorname{dim}_{F} \operatorname{graph} r,
$$

for both $\operatorname{dim}_{F}=\operatorname{dim}_{H}$ and $\operatorname{dim}_{F}=\operatorname{dim}_{M}$ (for $\operatorname{dim}_{H}$ this was also shown in Theorem 1 of [13]). We conclude by Theorem 3.4 that $A$ is a fractal star body with respect to $\operatorname{dim}_{F}$ for both of these dimensions. Moreover, since the graph of the function $r$ is locally fractal with respect to both $\operatorname{dim}_{M}$ and $\operatorname{dim}_{H}$, the same is true for the graph of $\tilde{r}$, and therefore $A$ is a locally fractal star body with respect to both $\operatorname{dim}_{M}$ and $\operatorname{dim}_{H}$.

It is clear that if we replace $\sin$ in (15) by cos, we obtain fractal star bodies with the same dimensions. However, the shapes can be fairly distinct as some of our examples show. Figures 1, 2, 3, 4, and 5 show examples of these star bodies for different values of $\lambda$, and $s$, as indicated. In Figure 1 we have taken $\gamma=0$; in all the other figures we have taken $\gamma=1$.
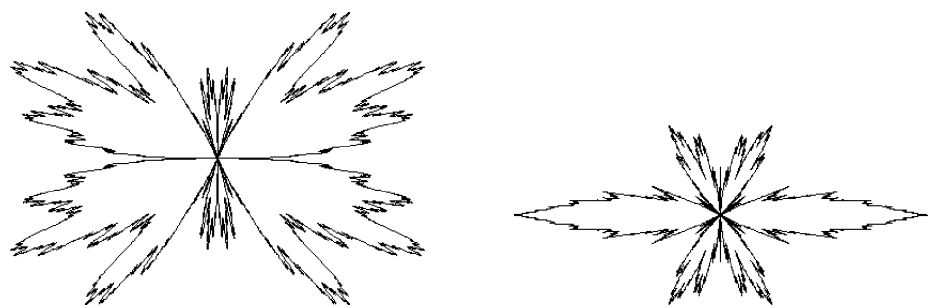

Fig. 1. Fractal star bodies in $\tilde{\mathcal{S}}^{2}$ derived from the Weierstrass function with $\lambda=2$, and $s=1.3$. We used the sin function for the left body and the cos function for the right body.
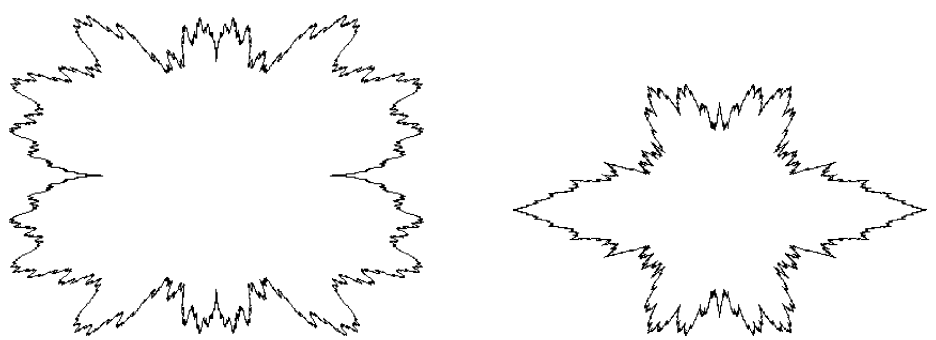

Fig. 2. Fractal star bodies in $\mathcal{S}^{2}$ derived from the Weierstrass function with $\lambda=2$, and $s=1.3$. We used the sin function for the left body and the cos function for the right body. 

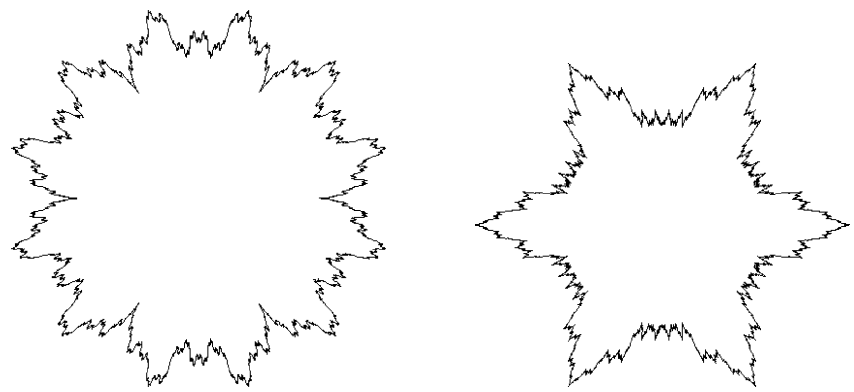

Fig. 3. Fractal star bodies in $\mathcal{S}^{2}$ derived from the Weierstrass function with $\lambda=3$, and $s=1.3$. We used the sin function for the left body and the cos function for the right body.
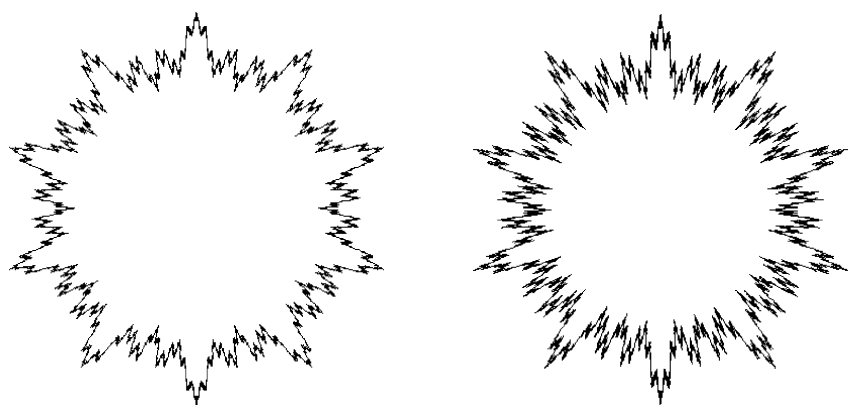

Fig. 4. Fractal star bodies in $\mathcal{S}^{2}$ derived from the Weierstrass function with $\lambda=5 ; s=1.4$ for the left body and $s=1.5$ for the right body.
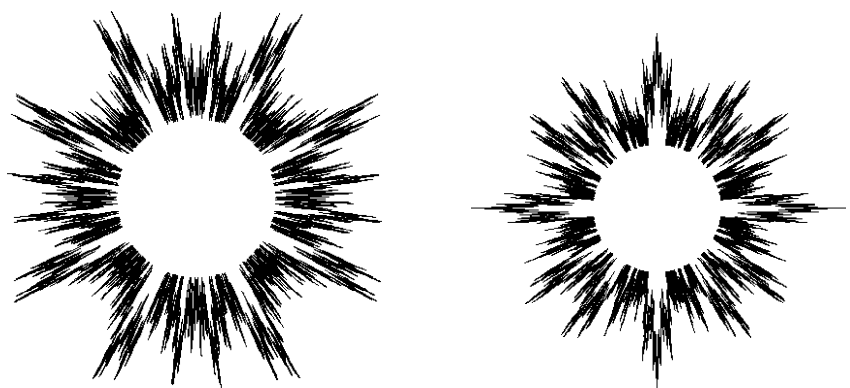

Fig. 5. Fractal star bodies in $\mathcal{S}^{2}$ derived from the Weierstrass function with $\lambda=4$, and $s=1.7$. We used the sin function for the left body and the cos function for the right body.

4. Operations on fractal star bodies. Let $\mathcal{F S}^{n}\left(\operatorname{dim}_{F}\right)$ be the subfamily of $\mathcal{S}^{n}$ with members that are fractal with respect to $\operatorname{dim}_{F}$. Similarly, let $\mathcal{F} \tilde{\mathcal{S}}^{n}\left(\operatorname{dim}_{F}\right)$ be the subfamily of $\tilde{\mathcal{S}}^{n}$ with members that are fractal with respect to $\operatorname{dim}_{F}$.

It is natural to look for possible operations on $\mathcal{S}^{n}$ or $\tilde{\mathcal{S}}^{n}$ which preserve

$$
\mathcal{F S}^{n}\left(\operatorname{dim}_{F}\right), \mathcal{F S}^{n}\left(\operatorname{dim}_{H}\right) \text { or } \mathcal{F S}^{n}\left(\operatorname{dim}_{M}\right),
$$

or the corresponding extended families. 


\section{Transformations of the radial function}

Definition 4.1. A continuous function

$$
\phi:(0, \infty) \rightarrow(0, \infty)
$$

$($ or $\phi:[0, \infty) \rightarrow[0, \infty)$ with $\phi(0)=0)$ gives rise to a function

$$
F_{\phi}: \mathcal{S}^{n} \rightarrow \mathcal{S}^{n}
$$

(or, $\tilde{F}_{\phi}: \tilde{\mathcal{S}} \rightarrow \tilde{\mathcal{S}}$, respectively), defined for any $A \in \mathcal{S}^{n}$ (or $A \in \tilde{\mathcal{S}}^{n}$, respectively) by

$$
F_{\phi}(A)=A(\phi), \quad \text { where } \rho_{A(\phi)}(u):=\phi\left(\rho_{A}(u)\right) \text { for } u \in S^{n-1} .
$$

THEOREM 4.2. (i) If $\phi:(0, \infty) \rightarrow(0, \infty)$ is bilipschitz, then for every fractal dimension $\operatorname{dim}_{F}$, the function $F_{\phi}$ is a unary operation on the family $\mathcal{F} \mathcal{S}^{n}\left(\operatorname{dim}_{F}\right)$.

(ii) If $\phi:[0, \infty) \rightarrow[0, \infty)$ is bilipschitz and $\phi(0)=0$, then for every fractal dimension $\operatorname{dim}_{F}$, the function $\tilde{F}_{\phi}$ is a unary operation on $\mathcal{F} \tilde{\mathcal{S}}^{n}\left(\operatorname{dim}_{F}\right)$.

Proof. For Part (i), it suffices to prove that $A(\phi) \in \mathcal{F S}^{n}\left(\operatorname{dim}_{F}\right)$. Evidently, $\rho_{A(\phi)}(u)>0$ for all $u \in S^{n-1}$, that is, $A(\phi) \in \mathcal{S}^{n}$. Further, the function $g_{\phi}: \operatorname{graph} \rho_{A} \rightarrow \operatorname{graph} \rho_{A(\phi)}$ defined by

$$
g_{\phi}\left(u, \rho_{A}(u)\right):=\left(u, \phi \rho_{A}(u)\right)
$$

is bilipschitz as the Cartesian product of $\operatorname{id}_{S^{n-1}}$ and $\phi \mid \rho_{A}\left(S^{n-1}\right)$. Thus,

$$
\operatorname{dim}_{F} \operatorname{graph} \rho_{A(\phi)}=\operatorname{dim}_{F} \operatorname{graph} \rho_{A} .
$$

For Part (ii), note that $\phi$ is a bijection and $\phi(0)=0$. It follows that

$$
\left(\rho_{A(\phi)}\right)^{-1}(0)=\rho_{A}^{-1}(0) .
$$

Evidently, graph $\left(\rho_{A} \mid \rho_{A}^{-1}(0)\right)$ is a subset of $S^{n-1} \times\{0\} \subset S^{n-1} \times \mathbb{R}$. Therefore, it has fractal dimension less than or equal to $n-1$. The same holds for $A(\phi)$. Moreover, since clint $A=A$, there is a connected subset $T$ of $S^{n-1}$ with a non-empty interior such that $\rho_{A}(T) \subseteq(0, \infty)$ and consequently, $\rho_{A(\phi)}(T) \subseteq(0, \infty)$.

Hence, by Axiom F.2,

$$
\operatorname{dim}_{F} \operatorname{graph} \rho_{A(\phi)}=\operatorname{dim}_{F} \operatorname{graph}\left(\rho_{A(\phi)} \mid\left(S^{n-1} \backslash\left(\rho_{A(\phi)}\right)^{-1}(0)\right),\right.
$$

and

$$
\operatorname{dim}_{F} \operatorname{graph} \rho_{A}=\operatorname{dim}_{F} \operatorname{graph}\left(\rho_{A} \mid\left(S^{n-1} \backslash \rho_{A}^{-1}(0)\right)\right) .
$$

Since the right hand sides are equal because $\phi$ is bilipschitz, the left hand sides are equal too.

As an example of such a function we consider star duality (see [14], [15]). Let $i: \mathbb{R}^{n} \backslash$ $\{0\} \rightarrow \mathbb{R}^{n} \backslash\{0\}$ be the inversion with respect to $S^{n-1}$ :

$$
i(x):=\frac{x}{\|x\|^{2}} .
$$

For every $A \in \mathcal{S}^{n}$, the star dual $A^{\circ}$ is defined by

$$
A^{\circ}:=\operatorname{cl}\left(\mathbb{R}^{n} \backslash i(A)\right) \text {. }
$$

Then

$$
\rho_{A^{\circ}}=\frac{1}{\rho_{A}} .
$$


THEOREM 4.3. Star duality preserves the family $\mathcal{F S}^{n}\left(\operatorname{dim}_{F}\right)$ for every fractal dimension $\operatorname{dim}_{F}$.

Proof. Let $A \in \mathcal{S}$. We will show that $\operatorname{dim}_{F}$ of the boundary of $A$ is the same as $\operatorname{dim}_{F}$ of the boundary of its star dual $A^{\circ}$. Since $A$ is compact and $\rho_{A}$ is positive, it follows that there exists a constant $\alpha \geq 1$ such that $\rho_{A}(u) \in\left[\frac{1}{\alpha}, \alpha\right]$ for every $u \in S^{n-1}$.

Let $\phi(t):=\frac{1}{t}$ for $\frac{1}{\alpha} \leq t \leq \alpha$. Then $A^{\circ}=A(\phi)$. By Theorem 4.2 (i) it suffices to prove that the function $\phi$ is bilipschitz. By easy calculation, $\phi$ is a Lipschitz function. Since $\phi$ is an involution, it is bilipschitz.

Radial sums. For any two star bodies $A, B \in \mathbb{R}^{n}$ the radial sum $A \tilde{+} B$ is determined by its radial function:

$$
\rho_{A \tilde{+} B}=\rho_{A}+\rho_{B}
$$

(compare [7], p. 19, and [15], p. 177).

TheOREM 4.4. Let $L \in \tilde{\mathcal{S}}^{n}$. If $\rho_{L}$ is a Lipschitz function, then for every $A$

$$
\operatorname{dim}_{H} \operatorname{graph} \rho_{A \tilde{+} L}=\operatorname{dim}_{H} \operatorname{graph} \rho_{A} .
$$

Proof. Let $X:=X_{n}$ and $p:=p_{n}$ (see (5)-(7)). Consider the subsets $X_{n}(k, \varepsilon)$ defined in (8) and (9) for $k \in\{0,1\}^{n-1}$ and $\varepsilon \in\left(0, \frac{\pi}{4}\right)$. Further, let $r_{A}=\rho_{A} \circ p$ and $r_{L}=\rho_{L} \circ p$ (see (12)). Then, by Lemma 3.2, $r_{L} \mid X(k, \varepsilon)$ is a Lipschitz function for $k$ and $\varepsilon$ as above, whence by Theorem 1 in [13],

$$
\operatorname{dim}_{H} \operatorname{graph}\left(r_{A \tilde{+} L} \mid X(k, \varepsilon)\right)=\operatorname{dim}_{H} \operatorname{graph}\left(r_{A} \mid X(k, \varepsilon)\right) .
$$

Thus, by Remark 3.3 and $\sigma$-stability of $\operatorname{dim}_{H}$,

$$
\begin{aligned}
\operatorname{dim}_{H} \operatorname{graph}\left(r_{A \tilde{+} L}\right) & =\sup _{k, \varepsilon} \operatorname{dim}_{H} \operatorname{graph}\left(r_{A \tilde{+} L} \mid X(k, \varepsilon)\right)=\sup _{k, \varepsilon} \operatorname{dim}_{H} \operatorname{graph}\left(r_{A} \mid X(k, \varepsilon)\right) \\
& =\operatorname{dim}_{H} \operatorname{graph} r_{A} .
\end{aligned}
$$

By Theorem 3.4, this implies equality (17).

Corollary 4.5. Let $L \in \tilde{\mathcal{S}}^{n}$. If $\rho_{L}$ is a Lipschitz function, then

$$
A \in \mathcal{F} \tilde{\mathcal{S}}^{n}\left(\operatorname{dim}_{H}\right) \Rightarrow A \tilde{+} L \in \mathcal{F} \tilde{\mathcal{S}}^{n}\left(\operatorname{dim}_{H}\right) .
$$

Corollary 4.5 combined with Lemma 3.10 in [20] yields the following.

Corollary 4.6. If $L \in \tilde{\mathcal{S}}^{n}$ and $0 \in \operatorname{int} \operatorname{ker} L$, then

$$
A \in \mathcal{F} \tilde{\mathcal{S}}^{n}\left(\operatorname{dim}_{H}\right) \Rightarrow A \tilde{+} L \in \mathcal{F} \tilde{\mathcal{S}}^{n}\left(\operatorname{dim}_{H}\right) .
$$

Quotient star bodies. The notion of quotient star body $A / C$ for $A, C \in \tilde{\mathcal{S}}^{n}$ and $C \subset \operatorname{int} A$ was introduced in [14] as a metric counterpart of the topological notion of quotient set. By Proposition 2.3 in [14],

$$
\rho_{A / C}=\rho_{A}-\rho_{C} .
$$

Theorem 4.4 and Corollaries 4.5 and 4.6 have their counterparts for quotient star bodies: Theorem 4.7 and Corollaries 4.8 and 4.9, respectively. The proofs are analogous to those for Theorem 4.4 and Corollaries 4.5 and 4.6 , we only replace $\rho_{L}$ by $-\rho_{C}$. 
TheOREM 4.7. Let $A, C \in \tilde{\mathcal{S}}^{n}$ and $C \subset \operatorname{int} A$. If $\rho_{C}$ is a Lipschitz function, then $\operatorname{dim}_{H} \operatorname{graph} \rho_{A / C}=\operatorname{dim}_{H} \operatorname{graph} \rho_{A}$.

Corollary 4.8. If $A, C \in \tilde{\mathcal{S}}^{n}, C \subset \operatorname{int} A$, and $\rho_{C}$ is a Lipschitz function, then

$$
A \in \mathcal{F} \tilde{\mathcal{S}}^{n}\left(\operatorname{dim}_{H}\right) \Rightarrow A / C \in \mathcal{F} \tilde{\mathcal{S}}^{n}\left(\operatorname{dim}_{H}\right) .
$$

Corollary 4.9. If $A, C \in \tilde{\mathcal{S}}^{n}$ and $0 \in \operatorname{int} \operatorname{ker} C$, then

$$
A \in \mathcal{F} \tilde{\mathcal{S}}^{n}\left(\operatorname{dim}_{H}\right) \Rightarrow A / C \in \mathcal{F} \tilde{\mathcal{S}}^{n}\left(\operatorname{dim}_{H}\right) .
$$

5. Cylinders and suspensions of star bodies. For any nonempty compact subset $A$ of the hyperplane $\left(e_{n}\right)^{\perp}$ in $\mathbb{R}^{n}$, we define the cylinder over $A$ by

$$
C(A):=A+\Delta\left(-e_{n}, e_{n}\right) .
$$

Evidently, $C(A)$ is isometric to the Cartesian product $A \times[-1,1]$.

TheOREM 5.1. If $A \in \mathcal{F} \tilde{\mathcal{S}}^{n-1}\left(\operatorname{dim}_{H}\right)$, then

$$
C(A) \in \mathcal{F} \tilde{\mathcal{S}}^{n}\left(\operatorname{dim}_{H}\right) .
$$

Also, the corresponding statement holds for the classes $\mathcal{F S}^{n-1}\left(\operatorname{dim}_{H}\right)$ and $\mathcal{F S}^{n}\left(\operatorname{dim}_{H}\right)$. Proof. By the assumption, $\operatorname{dim}_{H}$ bd $A>n-2$.

By Corollary 7.4 of the product formula in [5] it follows that

$$
\operatorname{dim}_{H} C(\mathrm{bd} A)=\operatorname{dim}_{H} \mathrm{bd} A+1 \text {. }
$$

But

$$
\operatorname{bd} C(A)=C(\operatorname{bd} A) \cup\left(A+\left\{-e_{n}, e_{n}\right\}\right) ;
$$

hence, by $(19), \operatorname{dim}_{H}$ bd $C(A) \geq \max \left\{\operatorname{dim}_{H}\right.$ bd $\left.A+1, n-1\right\}>n-1$. By Corollary 2.7 this implies (18).

If $n>2$ and $1 \leq k \leq n-2$, we can define the iteration $C^{(k)}: \mathcal{S}^{n-k} \rightarrow \mathcal{S}^{n}$ of $C$ :

$$
C^{(1)}:=C, C^{(k+1)}:=C \circ C^{(k)} .
$$

Then, evidently, $C^{(k)}(A)$ is isometric to the product of $A$ and $k$-dimensional cube. Thus, by (19),

$$
\operatorname{dim}_{H} \operatorname{bd} C^{(k)}(A)=\operatorname{dim}_{H} \operatorname{bd} A+k .
$$

Next, we want to consider the notion of a 'geometric suspension' (the geometric counterpart of the well known topological notion of suspension). We adopt the symbol $S A$ for this suspension of $A$.

For any nonempty compact subset $A$ of the hyperplane $\left(e_{n}\right)^{\perp}$ in $\mathbb{R}^{n}$ we define the suspension of $A$ to be the union of two cones over $A$ : for every $c \in \operatorname{lin}\left(e_{n}\right)$, let

$$
\operatorname{cone}(A, c):=\bigcup\{\Delta(x, c) \mid x \in A\} ;
$$

then the suspension of $A$ is defined by

$$
S A:=\operatorname{cone}\left(A, e_{n}\right) \cup \operatorname{cone}\left(A,-e_{n}\right) .
$$

In what follows, we identify $\left(e_{n}\right)^{\perp}$ with $\mathbb{R}^{n-1}$. Evidently,

$$
A \in \mathcal{S}^{n-1} \Rightarrow S A \in \mathcal{S}^{n}
$$


and the corresponding implication holds for $\tilde{\mathcal{S}}^{n}$. We shall prove the following.

THEOREM 5.2. Let $n \geq 3$. If $A \in \mathcal{F} \tilde{\mathcal{S}}^{n-1}\left(\operatorname{dim}_{H}\right)$, then

$$
S A \in \mathcal{F} \tilde{\mathcal{S}}^{n}\left(\operatorname{dim}_{H}\right) .
$$

The corresponding statement holds for the classes $\mathcal{F S}^{n-1}\left(\operatorname{dim}_{H}\right)$ and $\mathcal{F S}^{n}\left(\operatorname{dim}_{H}\right)$.

Proof. By (22),

$$
\operatorname{bd}(S A)=\operatorname{cone}\left(\operatorname{bd} A, e_{n}\right) \cup \operatorname{cone}\left(\operatorname{bd} A,-e_{n}\right)=S(\operatorname{bd} A) .
$$

For any natural number $k$, we define the subset $S_{k}$ of bd $(S A)$ to be

$$
S_{k}:=\operatorname{bd}(S A) \backslash\left(\left(e_{n}+\frac{1}{k} B^{n}\right) \cup\left(-e_{n}+\frac{1}{k} B^{n}\right)\right)
$$

and the subset $C_{k}$ of $C(\mathrm{bd} A)$,

$$
C_{k}:=\operatorname{bd} A+\operatorname{relint} \Delta\left(\left(1-\frac{1}{k}\right)\left(-e_{n}\right),\left(1-\frac{1}{k}\right) e_{n}\right) .
$$

It is easy to show that $S_{k}$ is bilipschitz equivalent to $C_{k}$, and thus, by Axiom F.1, for every $k$

$$
\operatorname{dim}_{H} S_{k}=\operatorname{dim}_{H} C_{k}
$$

Note that

$$
\operatorname{dim}_{H}(\operatorname{bd}(S A))=\operatorname{dim}_{H} \bigcup_{k \geq 3} S_{k}
$$

and similarly,

$$
\operatorname{dim}_{H}(C(\operatorname{bd} A))=\operatorname{dim}_{H} \bigcup_{k \geq 3} C_{k},
$$

because the Hausdorff dimension of the removed parts of the sets $S(\mathrm{bd} A)$ and $C(\mathrm{bd} A)$ is smaller ( 0 and at most $n-1$, respectively). Since the Hausdorff dimension is $\sigma$-stable, by (23) and (19) it follows that

$$
\operatorname{dim}_{H} \operatorname{bd}(S A)=\operatorname{dim}_{H} C(\operatorname{bd} A)=\operatorname{dim}_{H} \operatorname{bd} A+1>n .
$$

This completes the proof.

6. Kernels of fractal star bodies. The problem of characterizing star bodies with kernels of a given dimension was studied, for instance, in [6], [2], and [3]. In this section we will discuss what values the dimension of the kernel of a fractal star body can take.

We shall prove that the topological dimension of ker $A$ for a star body $A \in \mathcal{F}^{n}\left(\operatorname{dim}_{F}\right)$ is

- less than or equal to $n-1$ for arbitrary $\operatorname{dim}_{F}$;

- less than or equal to $n-2$ if $\operatorname{dim}_{F}$ is $\sigma$-stable.

We begin with the following.

TheOREm 6.1. Let $A$ be a star body in $\mathbb{R}^{n}$. Then, for arbitrary fractal dimension $\operatorname{dim}_{F}$, $\operatorname{dim} \operatorname{ker} A=n \Rightarrow \operatorname{dim}_{F} \operatorname{bd} A=n-1$. 
Proof. Let $a \in \operatorname{int} \operatorname{ker} A$, and let $A-a$ be the translated star body. Then by Lemma 2.19 in [20], the radial function $\rho_{A-a}$ is a Lipschitz map. Let $g:=\operatorname{id}_{S^{n-1}} \cdot \rho_{A-a}$, that is, $g(u)=u \rho_{A-a}(u)$ for every $u \in S^{n-1}$. By Lemma 2.3, $g$ is a Lipschitz map from $S^{n-1}$ onto bd $(A-a)$, and thus, by Axiom F.1 and Axiom F.3,

$$
\operatorname{dim}_{F} \operatorname{bd} A=\operatorname{dim}_{F} \operatorname{bd}(A-a) \leq \operatorname{dim}_{F} S^{n-1}=n-1,
$$

for arbitrary fractal dimension $\operatorname{dim}_{F}$. Hence, by Axiom F.0, $\operatorname{dim}_{F}$ bd $A=n-1$.

As a direct consequence of Theorem 6.1 we obtain the following.

Corollary 6.2. If $A$ is a fractal star body, then $\operatorname{dim} \operatorname{ker} A \leq n-1$.

For $\sigma$-stable dimensions we have a stronger result.

Theorem 6.3. Let $A \in \tilde{\mathcal{S}}^{n}$. If $\operatorname{dim}_{F}$ is $\sigma$-stable, then

$$
\operatorname{dim} \operatorname{ker} A=n-1 \Rightarrow \operatorname{dim}_{F} \operatorname{bd} A=n-1 .
$$

Proof. Let $H=\operatorname{aff} \operatorname{ker} A$ and let $E^{+}, E^{-}$be two closed half-spaces with $E^{+} \cup E^{-}=\mathbb{R}^{n}$ and $E^{+} \cap E^{-}=H$. There are two cases to be considered: when $\operatorname{ker} A \subset \operatorname{bd} A$, and when ker $A \cap \operatorname{int} A \neq \emptyset$. We will first consider the former and then show how the latter may be reduced to the former.

Case 1: Let $\operatorname{ker} A \subset \operatorname{bd} A$. Then $A$ is contained in one of the two half-spaces, because it is star-shaped with respect to every point of ker $A$. Assume $A \subset E^{+}$.

Let $a \in \operatorname{relint} \operatorname{ker} A$. Then there exists a ball $B$ with center $a$ such that $B \cap E^{+} \subset A$. We may assume that $a=0, H=\left(e_{n}\right)^{\perp}$, and $B=B^{n}$, because otherwise we can replace $A$ by a suitable homothet of $A$, whose properties involved in our statement will be the same as those of $A$. Also, let

$$
d:=\sup \{\|x\| \mid x \in A\} .
$$

Consider a sequence $\left(\phi_{k}\right)_{k \in \mathbb{N}}$ in $\left(0, \frac{\pi}{2}\right)$ with $\lim _{k \rightarrow \infty} \phi_{k}=\frac{\pi}{2}$, and the corresponding sequence $\left(T_{k}\right)_{k \in \mathbb{N}}$ of cones:

$$
T_{k}:=\left\{x \in E^{+} \mid \angle\left(x, e_{n}\right) \leq \phi_{k}\right\} .
$$

Let $C_{k}:=T_{k} \cap$ bd $A$ and $S_{k}:=T_{k} \cap$ bd $B$. We shall prove that for every $k$ the central projection $\xi_{k}: C_{k} \rightarrow S_{k}$ defined by $\xi_{k}(x):=\frac{x}{\|x\|}$ is bilipschitz.

Evidently, $\xi_{k}$ is a restriction of the metric projection (the nearest point map) of $\mathbb{R}^{n}$ onto $B$. Thus, $\xi_{k}$ is a weak contraction (see [15], Theorem 3.3.4). Hence, it suffices to show that there exists $\beta_{k}>0$ such that

$$
\left\|\xi_{k}(x)-\xi_{k}(y)\right\| \geq \beta_{k}\|x-y\| \text { for every } x, y \in C_{k} .
$$

For any $x \in \operatorname{bd} A$, let $\gamma(x):=\inf \{\angle(-x, b-x) \mid b \in H \cap \operatorname{bd} B\}$, and let

$$
\gamma_{k}:=\inf \left\{\gamma(x) \mid x \in C_{k}\right\} \text {. }
$$

Note that for any $b \in H \cap \operatorname{bd} B$, we have also $-b \in H \cap \operatorname{bd} B$ and the plane through $b, 0$ and $x$, contains the triangle $\Delta(-b, x, b)$. Note that one of its angles is $\angle(-b-x, b-x)=$ $\angle(-x, b-x)+\angle(-x,-b-x)$, so at least one of them is less than $\frac{\pi}{2}$. We conclude that $\gamma_{k}<\frac{\pi}{2}$ 
To prove that there is a value for $\beta_{k}$ such that (25) holds, take $x, y \in C_{k}$ and let $x^{\prime}=\xi_{k}(x), y^{\prime}=\xi_{k}(y)$. Let $\psi:=\angle(x, y)$. It is clear that $\angle\left(x^{\prime}, y^{\prime}\right)=\psi$ and $\psi \leq 2 \phi_{k}<\pi$. We may assume that $x \neq y$ and $\|x\| \geq\|y\|$. Let now $y^{\prime \prime} \in \operatorname{pos} y \cap \operatorname{conv}(\{x\} \cup H \cap \operatorname{bd} B)$. Since $y \in \operatorname{bd} A$, it follows that $y^{\prime \prime} \in \Delta(0, y)$.

If

$$
\psi \geq \frac{\pi}{2}-\gamma_{k}
$$

then

$$
\left\|x^{\prime}-y^{\prime}\right\|=2 \sin \frac{\psi}{2} \geq \sin \left(\frac{\pi}{4}-\frac{\gamma_{k}}{2}\right) \cdot d^{-1}\|x-y\|,
$$

since $\left\|x^{\prime}\right\|=\left\|y^{\prime}\right\|=1$. So in this case we may take $\beta_{k}:=\sin \left(\frac{\pi}{4}-\frac{\gamma_{k}}{2}\right) \cdot d^{-1}$.

Assume now that

$$
\psi<\frac{\pi}{2}-\gamma_{k}
$$

Let us consider the triangle $\Delta(x, 0, y)$ and let $\delta:=\angle(x-y,-y)$. If $\delta \geq \frac{\pi}{2}$, then

$$
\left\|x-y^{\prime \prime}\right\| \geq\|x-y\|
$$

since $\Delta\left(x, y^{\prime \prime}\right)$ is the longest side in the triangle $\Delta\left(x, y^{\prime \prime}, y\right)$. If $\delta<\frac{\pi}{2}$, then by the rule of sines for $\Delta\left(x, y^{\prime \prime}, y\right)$, the assumption that $\|x\| \geq\|y\|$, and (26),

$$
\begin{aligned}
\left\|x-y^{\prime \prime}\right\| & =\frac{\|x-y\| \sin \delta}{\sin \left(\angle\left(y^{\prime \prime}-y, y^{\prime \prime}-x\right)\right)} \geq\|x-y\| \sin \delta \\
& >\|x-y\| \sin \frac{\pi-\psi}{2}>\|x-y\| \sin \left(\frac{\pi}{4}+\frac{\gamma_{k}}{2}\right) .
\end{aligned}
$$

Therefore, by (27) and (28), we obtain $\left\|x-y^{\prime \prime}\right\| \geq c_{k}\|x-y\|$ for $c_{k}=\sin \left(\frac{\pi}{4}+\frac{\gamma_{k}}{2}\right)$.

Moreover, by the rule of sines for $\Delta\left(x, 0, y^{\prime \prime}\right)$, the minimality of $\gamma_{k}$, and (24),

$$
\frac{\left\|x-y^{\prime \prime}\right\|}{\sin \psi}=\frac{\|x\|}{\sin \left(\psi+\angle\left(-x, y^{\prime \prime}-x\right)\right)} \leq \frac{d}{\sin \left(\psi+\gamma_{k}\right)} .
$$

Thus, by (26),

$$
\left\|x-y^{\prime \prime}\right\| \leq \frac{d}{\sin \gamma_{k}} \sin \psi
$$

whence

$$
\|x-y\| \leq \frac{d}{c_{k} \sin \gamma_{k}} \sin \psi
$$

Since

$$
\left\|x^{\prime}-y^{\prime}\right\|=2 \sin \frac{\psi}{2}=\frac{\sin \psi}{\cos \frac{\psi}{2}}
$$

by (29) it follows that

$$
\|x-y\| \leq \frac{d}{c_{k} \sin \gamma_{k}} \cos \frac{\psi}{2}\left\|x^{\prime}-y^{\prime}\right\| \leq \frac{d}{c_{k} \sin \gamma_{k}}\left\|x^{\prime}-y^{\prime}\right\| .
$$

Taking now $\beta_{k}:=\left(\frac{d}{c_{k} \sin \gamma_{k}}\right)^{-1}$, we obtain the inequality (25) required.

Thus for every $k$ the function $\xi_{k}$ is bilipschitz, whence by Axiom F.1 combined with Axioms F.0 and F.3,

$$
\operatorname{dim}_{F} C_{k}=\operatorname{dim}_{F} S_{k}=n-1 .
$$


Further, $\bigcup_{k \in \mathbb{N}} T_{k}=\{0\} \cup \operatorname{int} E^{+}$, whence

$$
\operatorname{bd} A=(H \cap \operatorname{bd} A) \cup \bigcup_{k \in \mathbb{N}} C_{k} .
$$

By (30), (31) and $\sigma$-stability of $\operatorname{dim}_{F}$, we infer that $\operatorname{dim}_{F} \operatorname{bd} A=n-1$.

Case 2: Let $\operatorname{ker} A \cap \operatorname{int} A \neq \emptyset$. Then $\operatorname{relint} \operatorname{ker} A \subset \operatorname{int} A$, because otherwise $A$ would not be star-shaped at points of relint ker $A \backslash \operatorname{int} A$. Thus, $H$ separates $A$ into two star bodies $A_{1}, A_{2}$ such that $\operatorname{ker} A_{1}=\operatorname{ker} A_{2} \subset \operatorname{bd} A_{i}$ for $i=1,2$. Then, as in Case 1 , $\operatorname{dim}_{F} \operatorname{bd} A_{i}=n-1$ for $i=1,2$. Since bd $A \cup(H \cap A)=\operatorname{bd} A_{1} \cup \operatorname{bd} A_{2}$, it follows from Axiom F.2 that $\max \left(\operatorname{dim}_{F}(\mathrm{bd} A, n-1)=n-1\right.$. Hence $\operatorname{dim}_{F} \mathrm{bd} A \leq n-1$, and thus $\operatorname{dim}_{F}$ bd $A=n-1$ by Axiom F.0.

Corollary 6.4. If $\operatorname{dim}_{F}$ is $\sigma$-stable and $A \in \mathcal{F} \tilde{\mathcal{S}}^{n}\left(\operatorname{dim}_{F}\right)$, then $\operatorname{dim} \operatorname{ker} A \leq n-2$.

The following simple example shows that for the Hausdorff dimension the upper bound in Corollary 6.4 cannot be decreased.

ExAmple 6.5. If $A_{0} \in \mathcal{F} \tilde{\mathcal{S}}^{2}\left(\operatorname{dim}_{H}\right)$, then by Corollary $6.4, \operatorname{dim} \operatorname{ker} A_{0}=0$ (such a star body $A_{0}$ exists, see Example 3.8 for instance).

For $n>2, A_{0}$ can be embedded isometrically in $\operatorname{lin}\left(e_{1}, e_{2}\right)$. Define

$$
A_{n}:=C^{(n-2)}\left(A_{0}\right),
$$

(as in (20)). Then $\operatorname{dim} \operatorname{ker} A_{n}=\operatorname{dim} \operatorname{ker} A_{0}+n-2=n-2$, because ker $A_{n}$ is the product of ker $A_{0}$ and an $(n-2)$-dimensional cube.

Hence, for every $n \geq 2$ there exists an $A_{n} \in \mathcal{F}^{n}\left(\operatorname{dim}_{H}\right)$, with $\operatorname{dim} \operatorname{ker} A_{n}=n-2$.

REMARK 6.6. It is not clear whether the condition in Corollary 6.4 that $\operatorname{dim}_{F}$ be $\sigma$-stable is essential. In order to prove that it is essential one would need to construct an example of a star body with $\operatorname{dim} \operatorname{ker} A=n-1$, for which the boundary is fractal with respect to $\operatorname{dim}_{M}$ for instance. Whether such fractal star bodies exist is still an open problem.

\section{References}

[1] W. L. Bloch, Fractal boundaries are not typical, Topology Appl. 154 (2007), 533-539.

[2] M. Breen, Sets in $R^{d}$ having (d-2)-dimensional kernels, Pacific J. Math. 75 (1978), 37-44.

[3] M. Breen, A Helly-type theorem for the dimension of the kernel of a star-shaped set, Proc. AMS 73 (1979), 233-236.

[4] R. Engelking, Theory of Dimensions, Finite and Infinite, Sigma Series in Pure Mathematics 10, Heldermann Verlag, Lemgo, 1995.

[5] K. J. Falconer, Fractal Geometry, Wiley, 1990.

[6] K. J. Falconer, The dimension of the convex kernel of a compact star-shaped set, Bull. LMS 9 (1979), 313-316.

[7] R. Gardner, Geometric Tomography, Cambridge University Press, 1995.

[8] P. M. Gruber, Baire categories in convexity, in: Handbook of Convex Geometry, Vol. B, P. M. Gruber and J. M. Wills (eds.), North-Holland, 1993, 1327-1346.

[9] I. Herburt, M. Moszyńska and K. Rudnik, On intrinsic invariance of fractal dimensions, Geometriae Dedicata 57 (1995), 1-10. 
[10] W. Hurewicz and H. Wallman, Dimension Theory, Princeton University Press, 1948.

[11] R. Małysz, The Minkowski dimension of the bivariate fractal interpolation surfaces, Chaos, Solitons and Fractals 27 (2006), 1147-1156.

[12] B. B. Mandelbrot, Fractals: Form, Chance, and Dimension, W. H. Freeman and Co., San Francisco, CA, 1977.

[13] R. D. Mauldin and S. C. Williams, On the Hausdorff dimension of some graphs, Trans. AMS 298 (1986), 793-803.

[14] M. Moszyńska, Quotient star bodies, intersection bodies, and star duality, J. Math. Anal. Appl. 232 (1999), 45-60.

[15] M. Moszyńska, Selected Topics in Convex Geometry, Birkhäuser, 2005.

[16] V. Robins, Towards computing homology from finite approximations, in: Proceedings of the 14th Summer Conference on General Topology and its Applications (Brookville, NY, 1999), Topology Proc. 24 (1999), 503-532.

[17] K. Rudnik, Self-similar metric inverse limits of invariant geometric inverse sequences, Topology Appl. 48 (1992), 1-17.

[18] R. Schneider, Convex Bodies: the Brunn-Minkowski Theory, Cambridge University Press, 1993.

[19] R. Schneider, Convex surfaces, curvature and surface area measures, in: Handbook of Convex Geometry, Volume A, P. M. Gruber and J. M. Wills (eds.), North-Holland, 1993, 273-299.

[20] G. Sójka, Topological and metric properties of the hyperspace of star bodies, Doctoral Dissertation, Warsaw University of Technology, 2003 (in Polish).

[21] T. D. Taylor, Homeomorphism classes of self-contacting symmetric binary fractal trees, Fractals 15 (2007), 9-25.

[22] T. Zamfirescu, Baire categories in convexity, Atti Sem. Mat. Fis. Univ. Modena 39 (1991), $139-164$ 
